\title{
eJRIEPS
}

Ejournal de la recherche sur l'intervention en éducation physique et sport

Hors-série $\mathrm{N}^{\circ} 2 \mid 2018$

À propos de certaines bases théoriques et pratiques des sports collectifs

\section{Pratique et théorie : le rôle des connaissances et des modèles}

Jean-Francis Gréhaigne

\section{(2) OpenEdition \\ 1 Journals}

Édition électronique

URL : http://journals.openedition.org/ejrieps/504

DOI : $10.4000 /$ ejrieps.504

ISSN : 2105-0821

Éditeur

ELLIADD

Référence électronique

Jean-Francis Gréhaigne, "Pratique et théorie : le rôle des connaissances et des modèles », eJRIEPS

[En ligne], Hors-série № 2 | 2018, mis en ligne le 01 juillet 2018, consulté le 01 août 2019. URL : http:// journals.openedition.org/ejrieps/504; DOI : 10.4000/ejrieps.504

La revue eJRIEPS est mise à disposition selon les termes de la Creative Commons Attribution 4.0 International License. 


\section{Pratique et théorie : le rôle des connaissances et des modèles}

Comme mentionné dans l'introduction, on se méfie des théories dans le monde des jeux sportifs collectifs. La plupart des joueurs et des acteurs pensent que les théories sont déconnectées de la réalité... et qu'elles ne présentent donc aucun intérêt pour eux. Pourtant, une théorie n'est rien d'autre que la formulation d'une relation entre une cause et un effet. Elle peut ainsi s'avérer particulièrement utile. Dans les jeux et sports collectifs, l'essentiel des apprentissages consiste à «apprendre à faire» en rapport avec des connaissances (ne serait-ce que le règlement). À ce propos, nous avions déjà écrit que les rapports entre pratique et théorie étaient compliqués parfois insolubles (Gréhaigne, 1992). De la simple application de théorie produite ailleurs à une extraction exclusive de connaissance issue de cette pratique, le champ d'investigation pour étudier le phénomène est large. L'expérience nous a aussi montré qu'il ne faut jamais penser qu'une «application » directe de savoirs théoriques issus de la recherche est envisageable. C'est pourquoi, les apports théoriques sont souvent décriés. Cependant Kurt Lewin (1935) ne disait-il pas : «Il n'y a rien de plus pratique qu'une bonne théorie »? Dans cette optique, on doit se méfier des concepts de recherche appliquée et de recherche fondamentale au bénéfice d'une recherche plus novatrice qui doit être une ouverture vers de nouvelles perspectives. D'un autre point de vue, l'étude approfondie de la pratique, mélange contrasté d'idées et d'actions complétées par l'expérience, les connaissances et les compétences motrices du joueur, constitue un objet de recherche difficile à cerner et à appréhender comme tel. La prise en compte de cet ensemble a inspiré les travaux de Schön (1994) sur la pratique réflexive car si d'une théorie ou de la pratique on pouvait déduire à coup sûr la bonne décision, il suffirait de former des joueurs applicationistes. Or, cela ne fonctionne pas. On pourrait parler de «connaissance » versus «action » ou «activité » mais cela ne change pas vraiment la difficulté car le fond des choses n'est pas un problème de terminologie, mais un problème épistémologique et philosophique.

Lorsque l'on débat à propos de «théories », on est dans une activité discursive et symbolique qui vise une formalisation et prend la forme de discours explicatifs en relation avec une distanciation vis à vis du réel. Si on se situe du côté de la «pratique », on est dans une activité comportementale, concrètement sur le pôle de l'effection. Aussi, pour les hommes de terrain, la théorie est généralement dévalorisée au profit de la pratique. Mais toute théorie n'est pas raisonnement pur. Il y a des synergies entre la capacité qu'a le joueur d'agir concrètement sur le jeu et son aptitude à le conceptualiser à l'aide de la pensée tactique. Cependant, souvent d'un point de vue théorique, les données sont considérées comme émergeant d'une perception directe du jeu. Ici on considérera que la subjectivité et la cognition du joueur restent un élément fondamental pour le bon fonctionnement 
d'une pensée tactique efficace. La théorie permet de construire un cadre de références pour l'analyse de la réalité du jeu car la pratique se nourrit de son dialogue avec cet aspect théorique pour mieux répondre aux réalités de l'affrontement.

Enfin, dans le football, les rapports théorie / pratique ont fréquemment été pollués par les rapports au pouvoir des différents protagonistes. Donc, des distanciations étant voulues et entretenues, le contentieux est situé ailleurs qu'au plan épistémologique. On doit noter également que le travail de l'entraîneur et celui du chercheur ne relèvent pas du tout de la même logique. Le chercheur lui ne subit pas le même rapport au temps et s'appuie sur des savoirs constitués par un corps de connaissances en s'inscrivant dans la durée pour le faire évoluer. Il n'a pas à gagner dimanche ou répondre aux attentes directes des joueurs ou des fans et peut consacrer son temps à produire des données fiables. L'entraîneur, lui, s'appuie en partie sur les savoirs de la pratique qui sont avant tout un instrument opératoire qui permet d'agir sur le réel pour assurer la réussite (Delbos et Jorion, 1984). Ce savoir est tiré de l'expérience des joueurs et des entraîneurs, hommes de la pratique engagés dans l'action. Ce type d'apprentissage dépend d'expériences qu'il est difficile, voire parfois impossible de conceptualiser. On parlera alors parfois d'un «apprentissage sur le tas » (Labiche, 1983). Déjà, Bourdieu (1980) rappelait que «passer du schème pratique au schéma théorique, construit après la bataille, du sens pratique au modèle théorique, (...), c'est, parfois, laisser échapper tout ce qui fait la réalité temporelle de la pratique en train de se faire » (p. 136). Enfin, du joueur de sport collectif, on accepte la part d'irrationnel inhérente à toute action humaine complexe. «En ce sens, on lui demande un rapport à la théorie qui ne soit pas révérencieux et dépendant mais au contraire critique, pragmatique, voire opportuniste » (Perrenoud, 1994, p. 45).

\section{Incorporation de connaissances et références théoriques}

Pourtant, face au système complexe que représente une rencontre de sport collectif, la tentation est souvent grande d'adopter une logique déductive et réductionniste. La simplification à valeur heuristique atteint pourtant, ici, ses limites. La première tâche consiste souvent à faire évoluer les questions vers une posture de chercheur, qui serait la condition première de production de connaissances valides sur les phénomènes observés. De la théorie vers la pratique, certes la validité interne de la recherche décroît car la capacité à contrôler des facteurs expérimentaux au laboratoire fournit des informations de bonne valeur sur la variable isolée. Dans la pratique, la validité externe s'accroît fournissant ainsi des données utilisables immédiatement dans le jeu (Christina,1987).

Dans ce cadre, David (2002) propose la notion de discipline outil, comme la psychologie, la sociologie ou autres disciplines, pour faciliter les recherches en didactique des APS. En effet, ces études ne peuvent fonctionner que sur un paradigme de la complexité en posant que l'enseignement, la formation, l'entraînement, le partage d'expérience et la pensée tactique, sont des objets 
fondamentalement systémiques et culturels. Toute décomposition entraîne une réduction et un appauvrissement qui tourne le dos à l'émergence de connaissances utiles à l'intervention. Ce type de transmission n'est pas un retour de la psychopédagogie des années 1970, ni une psychologie ou sociologie appliquée. Il s'agit, en fait, de l'incorporation de quelques emprunts théoriques, conceptuels, méthodologiques ou instrumentaux pris à une discipline pour développer l'exercice et enrichir la responsabilité des chercheurs vis à vis des contenus qu'ils soient d'enseignement, d'entraînement ou de formation. Le tout dans un domaine de recherche dont la problématique reste fondamentalement didactique et consiste en l'étude des processus de conception, de transmission et d'appropriation des contenus dans ce qu'ils ont de spécifiques en vue de les améliorer.

Aussi, pour éviter l'application directe et sans précaution de théories extérieures au domaine considéré, la notion d'incorporation de connaissances nous semble bien correspondre aux enjeux du problème (Figure 1).

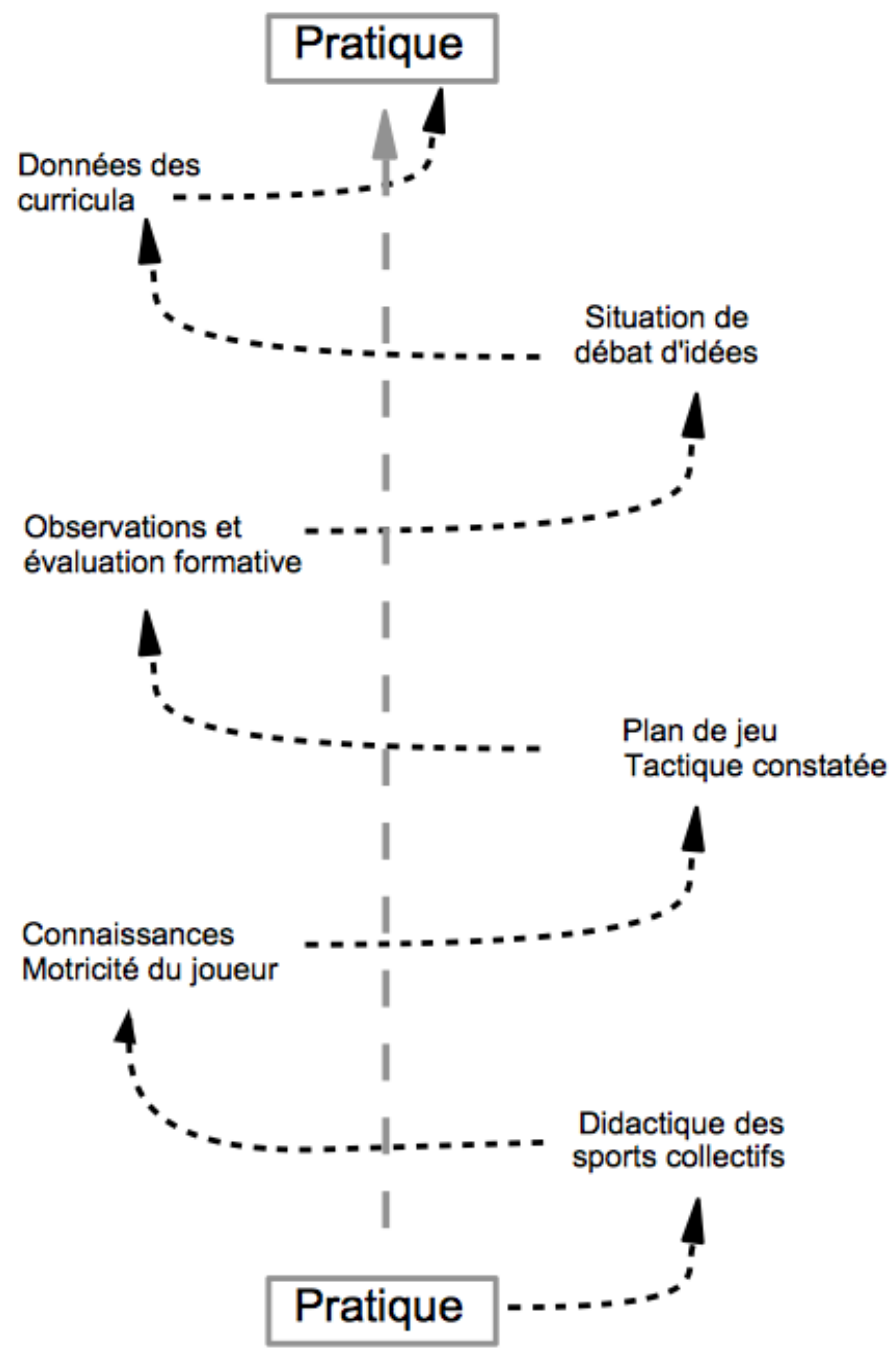

Figure 1. Incorporation de connaissances au cours de la pratique des jeux. 
S'approprier un objet de connaissance c'est le transformer et par là même se transformer soi-même. Ce double mouvement que l'on connaît bien dans le domaine de l'analyse des apprentissages en situation de travail (Pastré, 2011), est bien le signe de ce passage progressif d'un objet extérieur à l'objet intériorisé utilisable de façon crédible. Cela évite de se retrouver dans une logique déductive par rapport à des connaissances qui ont été produites dans d'autres champs et/ou d'autres circonstances et que l'on peut rarement appliquer aux situations de jeu. Donc, nous lui préfèrerons un modèle bâti selon un principe inductif (Gréhaigne \& Poggi, 2017). Utiliser des connaissances explicites à propos de la pratique, évaluer leur pertinence et retourner à la pratique pour apprécier leur à-propos nous paraît la voie la plus sûre pour éviter les errements d'une théorisation qui serait trop prescriptive de l'activité en jeu.

Dans le système enseignement / apprentissage, la première transformation consiste, à partir d'une éthologie de la pratique, à produire des programmes scolaires qu'on peut aussi dénommer curriculums formels ou prescrits. Chevallard (1985) appelle transposition didactique externe cette élaboration. La seconde transformation va des programmes aux contenus effectifs de l'enseignement. C'est la transposition interne qui relève largement de la marge d'interprétation des enseignants. Le troisième point concerne le processus de construction de ces savoirs et compétences par les élèves ce qui constitue une étape décisive dans leur parcours.

Toutefois, à propos de l'apologie de la pratique, il faut souligner que l'on n'apprend pas à jouer en faisant uniquement des jonglages avec la balle, en regardant les matches à la télévision ou en imitant les démonstrations sans faute d'un éducateur. L'apprentissage est une activité hautement plus complexe.

\section{Apport de la conscience de la situation}

La conceptualisation dans l'action cherche à montrer comment l'activité humaine est tout à la fois organisée, efficace, reproductible et analysable. Cela ne veut dire en aucune manière que c'est la théorie qui guide la pratique. Pour Pastré (2006), il y «a deux formes de la connaissance, qui correspondent à deux registres de conceptualisation. D'une part, il existe une forme prédicative ou discursive de la connaissance, qui s'exprime en énoncés et donne naissance à des savoirs ce qui correspond à un registre épistémique de conceptualisation : on énonce, dans un domaine, les objets, les propriétés et les relations qui le caractérisent. D’autre part, il existe une forme opératoire de la connaissance, qui correspond à un registre pragmatique de conceptualisation et qui a pour objectif d'orienter et de guider l'action » (Pastré, 2006, p. 4). C'est cette dernière forme opératoire de la connaissance qui sert quand il s'agit de faire une évaluation de la situation pour savoir comment décider et agir. Il y a de la conceptualisation dans les deux cas, dans un registre 
épistémique pour le premier mais aussi dans un registre pragmatique, ce qui est beaucoup moins avéré.

De ce point de vue, Ochanine (1978) fut le premier à distinguer ces deux registres. Si l'on suit ses propositions dans les situations de sport collectif à propos de l'activité du joueur, il existe, dans l'affrontement, des éléments et des relations qui, pour résoudre les problèmes posés par la situation de jeu, ne présentent pas d'intérêt. Le joueur peut en faire abstraction lorsqu'il tente de résoudre les problèmes posés par le jeu. Par contre, il existe d'autres relations indispensables pour faire évoluer favorablement les configurations du jeu. Ochanine (ibid) appelle image opérative la structure qui réunira toutes les relations indispensables à l'activité et elles seules. Cette structure sera, pour l'auteur, la plus pertinente, la plus fiable et donc la plus simple. L'auteur distingue les images opératives des images cognitives. Les images cognitives sont le reflet intégral des objets dans toute la diversité de leurs propriétés accessibles. En revanche, les images opératives sont des structures informationnelles spécialisées qui se forment au cours de telle ou telle action dirigée sur des objets (Ochanine \& Koslov, 1971). Il n'y a pas continuité entre l'image opérative d'une situation et l'image cognitive qui lui correspond, mais on peut parler d'articulation, selon des registres variés, entre des pôles différents mais toujours présents ensemble. Cette distinction permet aux auteurs de souligner l'ambivalence fonctionnelle de l'image : l'image est, en même temps, instrument de connaissance et régulateur de l'action. En effet, l'image opérative forme un ensemble informationnel spécialisé dont le contenu et la structure dépendent des tâches qui constituent une action concrète sur un objet. Comme chez les débutants, l'aspect cognitif tend à accumuler le plus d'informations possibles sur le jeu, ce qui est contradictoire à une décision rapide, alors que l'aspect opératif ne recense que les éléments utiles à la décision. Pour Gréhaigne (2011) dans une rencontre de sports collectifs, le traitement des configurations du jeu par le joueur expérimenté s'effectue par l'intermédiaire de ces images opératives. Ici, comme le joueur évolue dans un milieu dynamique et sur des objets dynamiques, le reflet de son activité sera également dynamique. De ce fait, pour Ochanine, Quaas et Zaltzman (1972), les images dynamiques correspondantes doivent être considérées comme des images opératives. De plus, l'aspect dynamique des images opératives correspond pour lui à une anticipation sur le devenir du processus contrôlé par le joueur. La prévision du déroulement des événements représente en fait leur reflet anticipé dans la conscience sous forme d'images dynamiques opératives. A la suite d'un tel reflet, l'image se transforme avec une certaine avance par rapport à la dynamique instantanée du jeu. Ce reflet anticipé n'est pas seulement cognitif. En effet, il permet au joueur de préparer ses actions, de mobiliser ses compétences motrices. Alors, l'image opérative n'est pas seulement le reflet subjectif du jeu, mais le reflet de l'action projetée sur les configurations du jeu en cours car, selon que la configuration du jeu sera en équilibre ou en déséquilibre transitoire, la conduite du joueur sera toute différente. 
Enfin, il est à noter que les images opératives ont tendance à rester dans l'implicite : la pratique va consister à construire une image opérative à partir de deux sources : l'image cognitive certes, mais aussi l'exercice de l'activité elle-même, avec les réussites ou les échecs qu'elle apporte. Dans l'autre cas, quand l'apprentissage se fait sur le tas, l'image opérative et l'image cognitive sont apprises en même temps, au point qu'il est difficile de les distinguer. Cette confusion est renforcée par le fait que, dans ces cas-là, l'image cognitive qui soutient l'image opérative est généralement de nature empirique. En définitive, c'est la réussite de l'action qui devient le critère de la pertinence de l'image cognitive empirique.

\section{Construction, déconstruction des connaissances et compétences}

Dans les sports collectifs, reste pour le joueur le problème de la mise à disposition et de la construction de ces images, et plus généralement des modèles théoriques. Pour résoudre ce problème, il faut bien souvent aider à l'identification des conceptions / représentations archaïques ou latentes chez les joueurs que dans Gréhaigne, Guillon et Mahut, (1995) nous avions dénommées «représentations conjoncturelles ». Ici, la représentation conjoncturelle est composée par ce qu'il y a de résiduel et de résistant dans les pratiques d'un sujet. Elle se caractérise par une mise en relation d'une représentation au sens de Richard «les représentations sont des constructions circonstancielles faites dans un contexte particulier à des fins spécifiques dans une situation donnée et pour faire face aux exigences de la tâche en cours » (Richard, 1990, p. 23) et de connaissances qui se trouvent dans la mémoire à long terme. Bien souvent ces connaissances ont été construites en dehors de toute volonté d'apprendre par «apprentissage fortuit » et fonctionnent chez le sujet sans que celui-ci en soit vraiment conscient. Elles sont donc extrêmement difficiles à faire évoluer car leur principale caractéristique est une résistance aux changements.

On constate aussi au cours de l'apprentissage, l'existence de compétences évidentes auxquelles succèdent de sérieux retours en arrière. Les processus d'apprentissage dans toute leur complexité reposent sur des acquisitions non linéaires. Pourquoi ? Sans doute parce que se développer, c'est aussi savoir inhiber une structure ou une notion concurrente. Le concept d'inhibition (Houdé, 1995) connait aujourd'hui un regain général d'intérêt en psychologie cognitive. Contrairement à Piaget, qui explique exclusivement le développement de l'intelligence par un mécanisme de coordination, on peut envisager qu'un autre mécanisme essentiel est aussi à l'œuvre : l'inhibition. Bref, le développement cognitif ne doit pas seulement être conçu comme l'acquisition progressive de connaissances, mais aussi comme relevant de la capacité d'inhibition de réactions ou de connaissances qui sont contradictoires avec la résolution des problèmes posés par les configurations du jeu à un moment donné. Bien souvent, en plus d'inhiber les réponses habituelles, il est nécessaire de les déconstruire car elles constituent un obstacle insurmontable à tout nouvel apprentissage. On 
peut ensuite reconstruire des réponses nouvelles qui, une fois stabilisées, prennent leur place dans le répertoire des réponses disponibles. Malléabilité et dynamisme semblent bien caractériser l'activité mentale et motrice des joueurs confrontés à la complexité du jeu.

Aussi nous visons, ici, un joueur praticien et agent de sa propre formation. Nous entendons par praticien, un joueur capable d'organiser le jeu mais également capable d'adapter, de réguler et d'évaluer ses actions face à des situations changeantes et évolutives. Cette notion de régulation nous semble essentielle. Elle comprend deux formes principales : l'une, au cours du jeu, qui consiste à ajuster ses actions ; 1'autre, a posteriori, qui vise à établir un bilan de ce qui s'est passé et à en tirer les conséquences. Au cours du jeu, la régulation tactique constitue un élément clef du dispositif car elle assure une adéquation permanente entre le système de contraintes et les ressources dont dispose le joueur. C'est au travers de cette mise en relation que s'expriment véritablement les présupposés de l'apprentissage qui sont les nôtres. Dans cette optique, un joueur concepteur et praticien capable d'analyser, de réguler sa pratique et de reformuler son projet devient, de ce fait, «agent de sa propre formation »(Lesnes, 1977, p. 178). Avoir pour perspective, un joueur agent de sa formation consiste à lui permettre d'établir une régulation entre sa pratique et des moments de réflexion / analyse sur celle-ci. Ainsi, c'est lui offrir l'occasion de gérer ses transformations en développant ses capacités d'observation et d'analyse (cf. le rôle de la vidéo dans l'analyse des prestations du joueur). Dès lors, le joueur peut construire des règles de gestion de son comportement qui lui permettront, éventuellement de poursuivre sa formation tout au long de sa carrière. La conception de la formation du joueur exposée ici permet de définir un cadre pour sa formation. Celui-ci se construit dans et par adaptation à un milieu contraignant : les rapports d'opposition.

\section{Conceptualisation, apprentissage et compétences motrices}

Par ailleurs, les compétences motrices sont un ensemble de manières d'agir, de gestes ou d'actions coordonnées qui présentent un caractère de rapidité et de stabilité. L'apprentissage de ces compétences motrices constitue un champ de recherche où le discours sur les éléments périphériques remplace, le plus souvent, de véritables connaissances sur comment cela fonctionne. Il est courant d'affirmer qu'afin d'acquérir les habiletés motrices, une grande quantité de pratique est nécessaire. A part cela et quelques broutilles, la suite reste encore largement inconnue. La variable la plus importante semble être la pratique : mais quelle pratique ? On a du mal à trier entre exécution à vide ou en situation, faire ses gammes, etc. De toute façon, il a toujours été affirmé que pour acquérir les habiletés motrices désirées, le joueur doit pratiquer de façon répétitive le même geste ou la même tâche. La tâche peut être divisée en ses composantes. Elle peut également être pratiquée de façon intensive ou alternée. Le retour d'informations influence les apprentissages moteurs et il s'agit d'un facteur important pour permettre l'acquisition d'habiletés motrices. 
Cette rétroaction peut être apportée sous plusieurs formes: verbale, démonstration totale ou partielle, analyse de postures. Dans les apprentissages moteurs, on vise une automatisation partielle des compétences motrices afin de dégager le canal cognitif conscient libéré pour assurer d'autres opérations. Cela consiste, par exemple, à faire exécuter, sauf alerte contraire au niveau des processus infra conscients, un certain nombre de compétences motrices pour offrir la possibilité de faire fonctionner d'autres programmes en même temps au niveau conscient.

Quant à elle, l'analyse conceptuelle des compétences motrices en jeu montre que les joueurs possèdent ou non un certain éventail de réponses motrices à leur disposition (celles de la vie courante ou d'autres beaucoup plus spécifiques et élaborées). Ces compétences reposent, nécessairement, sur un certain nombre de qualités physiques telles que la force ou la vitesse, avec comme fondement théorique, les «principes moteurs» (Gacon, 1984). En football, les principes moteurs sous-tendent le bon fonctionnement de ces compétences motrices. Ils sont organisés par le caractère spécifique des tensions musculaires ainsi que les postures et le placement des chaînes musculaires pour optimiser les réponses gestuelles. Il s'agit donc des compétences motrices construites par un joueur qui lui permettent, en jeu, l'appropriation, la conduite de balle et le tir. Il n'est pas question seulement de techniques réduites à ses composantes les plus apparentes (les gestes), d'ailleurs souvent travaillées à vide en-dehors des conditions réelles de l'activité. Il s'agit de tactique individuelle qui se traduit par une décision et une exécution sous contrôle d'un ou de plusieurs schèmes (Vergnaud, 2007) en situation d'affrontement. La tactique correspond à la mise en œuvre, à la coordination et à la régulation, à différents niveaux du contrôle moteur, de signaux envoyés aux muscles en vue de la réalisation du mouvement (Paillard, 1994). En retour, le joueur reçoit, durant l'exécution d'une tâche, un certain nombre d'informations de ses différents sens sur son placement, son déplacement, sa vitesse, ses tensions musculaires, les chocs éventuels, etc. Ces compétences motrices obéissent bien à des principes moteurs qui en conditionnent le fonctionnement.

Finalement, une description fine des compétences motrices demeure plus importante et féconde que toutes les explications à propos des théories non abouties au plan pratique sur l'apprentissage moteur. Néanmoins, si l'on peut parler de progrès dans les gestes, c'est bien que cet apprentissage évolue au fil du temps et des répétitions contextualisées, et qu'il représente lui-même un phénomène actif et évolutif. Ainsi, on peut dire que le geste, tout en étant « répété », est, à chaque utilisation en jeu, « reconfiguré » dans une certaine mesure, pouvant donc s'affiner mais aussi se détériorer ou se stéréotyper au fil des répétitions. L'aisance grandissante d'exécution d'un geste et son adaptation croissante au but visé sont le produit d'un travail d'apprentissage gestuel, lui-même intelligent, souvent en transformation progressive qu'il faut entretenir. Cependant, il importe aussi de ne jamais oublier que, sous le niveau de la commande consciente, il s'effectue une quantité importante de 
réglages automatisés fondamentaux, très complexes au niveau sensori-moteur et ceci de façon non consciente. Deleplace (1979, p. 11) propose, pour lier l'ensemble, la notion de «matrice tactique de l'exécution gestuelle » afin d'envisager les indispensables relations entre compétences motrices et tactiques en jeu. Ainsi il n'existe pas de technique générique isolée. Il n'existe que des techniques spécifiques que l'on choisit pour un cas donné. Néanmoins, cela implique que le joueur a effectivement à sa disposition un minimum de ressources motrices. Ainsi, toute activité en jeu est un acte forcément tactique, quel que soit le niveau où se situe le joueur, car il consiste à résoudre pratiquement, et dans le respect du règlement, un grand nombre de problèmes posés par les diverses situations de jeu. De la sorte, la technique que l'on qualifiera de personnelle représente des compétences motrices construites par le joueur et qui, dans un rapport de forces donné, constitue ses ressources face à une configuration du jeu à décoder, interpréter et faire évoluer. Dans ce cas, on parle bien de tactique individuelle pour bien souligner, la singularité du problème et le caractère indissociable des compétences motrices et de la pensée tactique pour résoudre le problème, le tout fonctionnant dans un temps contraint.

\section{Conceptualisation et modèle}

Dans la mobilisation et la conceptualisation des connaissances et des compétences motrices, on sous-estime encore trop souvent, la part de transformation que celles-ci doivent subir pour devenir des ressources. En sport collectif, le recours, entre autres, aux processus cognitifs conscients dans la gestion de certains aspects du jeu et de l'apprentissage a amené une profonde modification dans la gestion du jeu et des joueurs. Une conceptualisation s'effectue à partir du fonctionnement effectif de l'organisation du jeu. Aussi, cette meilleure connaissance d'une partie du réel permet-elle une gestion de l'affrontement mieux appropriée. Elle tend à élargir son assise au-delà des divisions traditionnelles des systèmes de jeu formel en lignes, en postes, couloirs, etc., pour intégrer et lui substituer des aspects plus dynamiques comme des trajets, des vitesses et des trajectoires de joueurs ainsi que du ballon. La conceptualisation est une forme d'activité imaginative qui permet de percevoir et d'interpréter les comportements des partenaires et des adversaires à partir des éléments caractéristiques principaux de l'image opérative du jeu : la finalisation, le laconisme et les déformations fonctionnelles (Ochanine et al., 1972).

Pour une meilleure efficacité, l'utilisation de théories ne saurait se concevoir aujourd'hui sans un recours à une présentation formalisée et inspirée des modèles conceptuels. L'objectif principal d'un modèle conceptuel est de transmettre les principes fondamentaux et les fonctionnalités de base du système qu'il représente. Une modélisation conceptuelle adaptée doit proposer de nouvelles perspectives et un ensemble d'outils nouveaux pour promouvoir le progrès dans le jeu mais forcément avec une marge d'erreurs. Par ailleurs, un modèle peut être défini comme un système 
figuratif reproduisant en partie la réalité sous forme schématisée, la rendant ainsi plus compréhensible. Le réel n'étant ni limpide ni directement accessible, il faut le décoder, le simplifier en ne conservant que les éléments et les relations qui semblent pertinents pour le cas traité. L'étude des évolutions de la pensée tactique en relation avec l'action incline à envisager, au moins dans les situations de jeu, des modifications, des dénaturations, des appauvrissements voire des enrichissements spectaculaires des connaissances et des compétences disponibles (Perrenoud, 2001). Ceci s'accomplit en relation avec une véritable transformation des observations et des prises d'information permises par la modélisation. Ce type d'opération très pragmatique met en perspective des évolutions et des transformations des connaissances et compétences motrices en vue de l'action. Certains de ces changements ne s'opèrent pas nécessairement dans la tête du joueur juste avant son action. Ils peuvent être incorporés à sa culture professionnelle qui véhicule un ensemble d'expériences, de méthodes, voire de «recettes» dont certaines résultent d'un changement ou d'une modification antérieure des connaissances et compétences.

Si la pratique demande des connaissances et des compétences motrices et, si l'on estime qu'il convient de les construire dans une perspective de formation, trois étapes semblent s'imposer :

- identifier et construire les ressources cognitives nécessaires, parmi lesquelles les connaissances ont leur importance ;

- entraîner à leur mobilisation en situation de jeu, à travers d'exercices, de situationsproblèmes, de cas à résoudre, etc. ;

- inscrire le tout dans une posture réflexive et professionnalisante, qui pousse le joueur à devenir acteur de ses transformations (Lesnes, 1977).

Nous présentons à la figure 2, un exemple de modélisation pour l'observation et l'analyse du jeu en mouvement en sports collectifs. Nous l'avons déjà en partie ébauché puis développé dans Gréhaigne et Godbout (2012, 2014). Notre ambition, avec cette idée de modélisation, correspond à l'action de production de modèles.

Le modèle envisagé décrit un certain nombre de faits à travers une structure explicative générale mais que l'on doit confronter à la réalité pour voir si elle reste pertinente, tout en fournissant une interprétation des rapports d'opposition faciles à comprendre pour les joueurs. Ce modèle est une construction, une structure que l'on peut utiliser comme référence, une sorte d'image analogique qui permet de matérialiser une idée, un concept ou une action (Gréhaigne, Marle, \& Zerai, 2013). Certaines configurations momentanées du jeu parmi les plus habituelles que l'on retrouve systématiquement dans le jeu des débutants ont été dénommées configurations «prototypiques »au sens où elles représentent un modèle original, archétype d'un modèle qui se reproduit. L'étude de l'ensemble des configurations prototypiques devrait permettre aux joueurs de construire des prototypes (cf. le concept d'images opératives, Ochanine, 1978) par catégorisation de formes, de 
relations temporelles et de classes de propriétés d'objets. On vise, ici, la mise à jour d'invariants pour reconnaître et décider vite à propos des configurations momentanées du jeu en vue d'être plus efficaces en jeu (Gréhaigne, 2007).

\begin{tabular}{|c|c|c|}
\hline $\begin{array}{c}\text { Avoir en tête pour } \\
\text { décider }\end{array}$ & $\begin{array}{l}\text { Dynamique d'organisation du jeu } \\
\text { (Les flèches indiquent les évolutions } \\
\text { potentielles dans la séquence de jeu) }\end{array}$ & Éléments observables \\
\hline $\begin{array}{l}\text { Références en arrière- } \\
\text { plan } \\
\text { - Matrices offensives et } \\
\text { défensives } \\
\text { - Configurations du jeu } \\
\text { prototypiques } \\
\text { - Ressources du joueur } \\
\text { - Réseau de compétences } \\
\text { - Consignes du coach } \\
\text { Conceptualisation } \\
\text { - Stratégie, plan de jeu } \\
\text { - Opportunités tactiques à } \\
\text { partir des éléments du jeu } \\
\text { - Score et déroulement de } \\
\text { la rencontre } \\
\text { - Connaissances } \\
\text { construites et ressources } \\
\text { disponibles }\end{array}$ & Avancer $\begin{array}{c}\text { Trajets et } \\
\text { trajectoires } \\
\text { du ballon }\end{array}$ & $\begin{array}{l}\text { Rapports d'opposition } \\
\text { - Positions du ballon } \\
\text { - Avance / Retard } \\
\text { - Barrage / Poursuite } \\
\text { - Position sur le terrain de } \\
\text { l'espace de jeu effectif } \\
\text { - Interpénétration et } \\
\text { positions des espaces des } \\
\text { EJEO et EJED } \\
\text { Jeu en mouvement } \\
\text { - Vitesses, accélérations, } \\
\text { trajets des joueurs } \\
\text { - Vitesses et variations } \\
\text { des trajectoires de balle } \\
\text { Observation formative } \\
\text { - Productions réelles et } \\
\text { démarches des joueurs }\end{array}$ \\
\hline & Défendre la cible & \\
\hline
\end{tabular}

Figure 2. Modélisation de la dynamique en sport collectif (adaptée de Gréhaigne \& Godbout, 2014)

La limite de ces modélisations vient du fait que les décisions des joueurs ne sont pas que rationnelles au sens où elles sont le fruit de l'interaction des autres composantes de l'action comme le réseau de compétences, le plan de jeu, le score, la fatigue. Bouthier (2000) met aussi en avant le fait que le référentiel commun des joueurs ne se réduit pas aux aspects décisionnels, mais intègre aussi l'expérience de ceux-ci dans ses aspects conceptuels certes, mais aussi moteurs, affectifs et sociaux.

D’une manière générale, pour les joueurs, « réussir » c'est marquer un but ou un point de plus que l'adversaire. Pour réaliser cet objectif, un des premiers principes consiste à créer un déséquilibre dans l'équipe adverse en prenant de l'avance sur son replacement défensif pour tenter de marquer 
un point. Si le déséquilibre n'existe pas, comment le faire apparaître ? Comme Gréhaigne et Godbout (2011) l'ont déjà mentionné, plusieurs stratégies sont possibles, soit :

- amener très vite le ballon en avant de l'espace de jeu effectif (EJE) ;

- récupérer la balle en avant de l'EJE ou au front du ballon ;

- attaquer dans la dimension momentanément faible de la défense ;

- attaquer dans la dimension forte de la défense pour la transformer.

Réussir ces actions suppose une circulation rapide du ballon et une judicieuse utilisation des espaces libres en relation avec un jeu en mouvement. Les transitions entre deux configurations du jeu apportent également de précieuses informations sur l'évolution du rapport de forces et permettent d'anticiper sur un replacement défensif en cours.

Avec cet ensemble de données, l'évolution du jeu peut alors se modéliser en concevant une évolution discontinue dans le temps. Pour ces situations complexes reposant sur des processus dynamiques, la modélisation en terme de boucle de régulation peut se complexifier de plusieurs façons. Par exemple, on peut introduire plusieurs boucles de régulation qui fonctionnent non plus de façon séquentielle mais en parallèle, d'où une certaine difficulté dans les rapports entre signes / reconnaissance / compréhension / exécution. La démarche repose essentiellement sur un concept d'activité référé à des possibilités anticipatrices et à des possibilités de réglages actifs (Piaget, 1974). Ici, action et réflexion sont définies comme étant intimement mêlées et se co-construisent en fonction d'un contexte de jeu donné.

\section{Conclusion}

Dans les formations théoriques qui ne prétendent pas préparer directement à une profession, l'ordre et le découpage des connaissances sont dictés par la cohérence des contenus et un souci de progression dans la complexité des connaissances et des compétences voire par les théories d'action en vogue (Lesnes, 1977). Dans la formation d'un joueur, il faut bien se préoccuper de la mobilisation de ces connaissances et des compétences motrices hors de l'enceinte du vestiaire, puisque, en sortant, le joueur est tout de suite confronté à une pratique sociale de référence (Martinand, 1981). Pour autant, la pratique reste saturée de données théoriques qu'elles soient conscientes ou non. Même si les apprentissages reposent sur un va-et-vient continuel entre affrontement, image du jeu et décision, il n'en reste pas moins que la concurrence entre expérience et connaissance est, aussi ici, bien réelle. Tout le problème pour la formation des joueurs est d'articuler ces composantes inséparables sans occulter la part irréductible due à une certaine nontransparence des pratiques et des habitus (Lenoir \& Pastré, 2008). Enfin, à la suite de Vygotsky (1933), on peut mettre au centre de l'analyse le caractère dynamique et évolutif des compétences motrices et des connaissances. Il faut alors envisager que les relations entre apprentissage et 
développement nécessitent d'examiner les relations entre quatre termes indissociablement liés : apprentissage, développement, expérience et maturation. Un joueur compétent doit disposer d'une part d'un ensemble de ressources cognitives parmi lesquelles des connaissances, des savoir-faire, des compétences motrices, des informations, des règles, des souvenirs de ses expériences, etc. Et d'autre part, le joueur doit avoir la capacité de les sélectionner, de les activer, de les coordonner, voire de les dépasser en situation, ce que Le Boterf (2000) met globalement sous l'étiquette de «mobilisation des ressources ».

Apprendre à faire, c'est apprendre par et dans l'activité en relation avec ses modèles. C'est probablement la forme première et la plus fondamentale d'apprentissage chez les humains. L'approche sémio-socio-constructiviste des apprentissages met l'accent sur l'activité du sujet et sa verbalisation pour appréhender ces phénomènes. C'est l'idée d'un sujet actif qui construit de nouvelles compétences ou connaissances reposant sur celles déjà en place. La compréhension s'élabore à partir des représentations que le sujet possède déjà. Dans cette perspective, on parlera de restructuration des informations en regard des conceptions / représentations particulières de chaque joueur. Processus qui apparaît profondément individuel, ce qui n'est pas sans poser problème pour la cohésion d'un groupe ou d'une équipe.

\section{Bibliographie}

Bourdieu, P. (1980). Le sens pratique. Paris : Les Éditions de Minuit.

Bouthier, D. (2000). La coordination des décisions individuelles ; contribution de l'intelligence tactique. Colloque Préparation Olympique L'évolution de la pensée tactique, Noisy le Grand, 14 et 15 novembre 2000.

Chevallard, Y. (1985) La transposition didactique. Du savoir savant au savoir enseigné. Grenoble : La Pensée Sauvage.

Christina, R. W. (1987). Motor learning: Future lines of research. In M. J. Safrit \& H. M. Eckert (Eds.), The cutting edge in physical education and exercise science research (American Academy of Physical Education Papers No. 20, pp. 26-41). Champaign, IL: Human Kinetics.

David, B. (2002). La psychologie comme discipline outil dans les recherches en didactique des APS. In B.David, \& J. Colomb, J. (Eds.) Impulsion 3. Recherche en didactique des activités physiques et sportives et en éducation physique et sportive (pp. 07-09).Paris : INRP.

Delbos, G., \& Jorion. (1984). La transmission des savoirs. Paris : Editions de la Maison des sciences de l'homme. 
Deleplace, R. (1979). Rugby de mouvement - Rugby total. Paris : Éducation Physique et Sport.

Gacon, G. (1984). La course d'endurance. Dijon : CRDP.

Gréhaigne, J.-F. (1992). L'organisation du jeu en football. Paris : ACTIO.

Gréhaigne, J.-F. (Ed.). (2007). Configurations du jeu, débat d'idées et apprentissage des sports collectifs. Besançon : Presses de l'Université de Franche-Comté.

Gréhaigne, J.-F. (2011). Quelques fondements théoriques à propos de l'analyse et de la perception du jeu. In J.-F. Gréhaigne (Ed.) Des signes au sens. Le jeu, les indices, les postures et les apprentissages dans les sports collectifs à l'école (pp. 21-38). Besançon: Presses de l'Université de Franche-Comté.

Gréhaigne, J.-F., \& Godbout, P. (2012). À propos de la dynamique du jeu ... en football et autres sports collectifs. eJRIEPS, 26, 130-156.

Gréhaigne, J.-F., \& Godbout, P. (2014). Dynamic systems theory and team sport coaching Quest, 66 (1), 96-116.

Gréhaigne, J-F., \& Poggi, M-P. (2017). Les connaissances et les compétences motrices en éducation physique : l'exemple des sports collectifs. In J. Moniotte (Ed.) Réussir l'écrit 2 (pp. 191-204). Paris : Atlante

Gréhaigne, J.-F., Marle, P., \& Zerai, Z. (2013). Modèles, analyse qualitative et configurations prototypiques dans les sports collectifs. eJRIEPS, 30, 05-25

Gréhaigne, J.-F., Guillon, R., \& Mahut, N. (1995). Les représentations conjoncturelles et la formation des jeunes enseignants d'éducation physique. In C. Paré (Ed.), Mieux enseigner l'éducation physique. Pensez-y! (pp. 191-207). Trois Rivières : Université du Québec à Trois Rivières, Canada.

Houde, O. (1992). Catégorisation et développement cognitif. Paris : P.U.F.

Le Boterf, G. (2000). Construire les compétences individuelles et collectives. Paris : Éditions d'organisation.

Labiche, J. (1983). Auto-socio-transformation et transformation sur le tas. D.I.R.E. en APS, 5, 1220

Lesnes, M. (1977). Travail pédagogique et formation d'adulte : éléments d'analyse. Paris : PUF.

Lenoir, Y., \& Pastré, P. (Eds.) (2008). Didactique professionnelle et didactiques disciplinaires en débat. Toulouse : Octarès.

Lewin, K. (1935). A dynamic theory of personality. New York : McGraw-Hill.

Martinand, J.L. (1981). Pratiques sociales de référence et compétences techniques. A propos d'un projet d'initiation aux techniques de fabrication mécanique en classe de quatrième. In A. Giordan (Ed.) Diffusion et appropriation du savoir scientifique : enseignement et 
vulgarisation (pp. 227-249) Actes des troisièmes journées Internationales sur l'Éducation Scientifique, Université Paris 7.

Ochanine, D. (1978). Le rôle des images opératives dans la régulation des activités de travail. Psychologie et Education, 2, 63-72.

Ochanine, D., \& Koslov, V. (1971). L'image opérative effectrice. Question de Psychologie, 3.

Ochanine, D., Quaas, W., \& Zaltzman, A. (1972). Déformation fonctionnelle des images opératives. Questions de Psychologie, 3.

Pastré, P. (2006). Apprendre à faire. In E Bourgeois \& G Chapelle, Apprendre et faire apprendre (pp. 109-121). Paris : PUF.

Pastré, P. (2011). La didactique professionnelle. Approche anthropologique du développement chez les adultes. Paris : PUF.

Perrenoud, P. (1994). Compétences, habitus et savoirs professionnels. European Journal of Teacher Education, 17, (1/2), 45-48.

Perrenoud, P. (2001). Les sciences de l'éducation proposent-elles des savoirs mobilisables dans l'action? Présentation orale aux Rencontres du Réseau Éducation et Formation, 10 au 12 avril 2001. Montréal : UQAM,.

Piaget, J. (1974). Réussir et comprendre. Paris : PUF.

Richard, J.-F. (1990). Les activités mentales. Comprendre, raisonner, trouver des solutions. Paris : Armand Colin.

Vygotski, L. (1933). Pensée et langage (traduction de Françoise Sève et avant-propos de Lucien Sève), suivi de «Commentaires sur les remarques critiques de Vygotski » de Jean Piaget, (Collection « Terrains», Éditions Sociales, Paris, 1985) ; Réédition, Paris : La Dispute, 1997. 